\title{
Incorporating Clinical Research into a Career in Gastroenterology and Hepatology
}

\author{
Robert J. Wong ${ }^{1}$
}

Published online: 22 September 2016

(c) Springer Science+Business Media New York 2016

\begin{abstract}
A former mentor once told me that there is a science and an art to clinical research. The science is the easy part-it can be taught and learned through education, mentoring, and experience. Yet, among medical practitioners, the art of clinical research is a less tangible concept, less easily articulated or imparted from mentor to mentee. It is a passion that fuels our enthusiasm, a spark that many of us find early in our careers. Nevertheless, the challenge as we transition from trainees, where time and resources to build upon this passion are more readily available, to attending physicians, is how to incorporate clinical research into one's early career. Although incorporating clinical research into a traditional academic career is generally straightforward, this pathway is not without challenges [1, 2]. Incorporating clinical research into a non-traditional environment is a far more daunting.
\end{abstract}

Similar to many gastroenterology fellows, I had the wonderful opportunity to train in a program (Stanford University Medical Center) that encouraged clinical research, providing ample resources, education, mentoring, and the protected time that enabled me and the other fellows to immerse themselves in research. A unique feature of the Stanford program was the ability to simultaneously complete a Master's degree in clinical epidemiology or health services research. While I had prior research experience before my fellowship years, the clinical epidemiology program provided an opportunity to truly understand

Robert J. Wong

rowong@alamedahealthsystem.org

1 Division of Gastroenterology and Hepatology, Alameda Health System - Highland Hospital, Highland Care Pavilion - 5th Floor, Endoscopy Unit, 1411 East 31st Street, Oakland, CA 94602, USA fundamental and advanced concepts of clinical research. Working in a multidisciplinary cohort to dissect our own research studies and to defend our choice of study design or statistical approach helped develop a skill set that helped ensure future success in clinical research. The ability to complete this program was truly instrumental in developing my career in clinical research.

After completing my gastroenterology fellowship, I joined the faculty at a Highland Hospital in Oakland, CA, a community-based teaching hospital that predominantly serves the indigent county population. My decision to join this practice stemmed from my underlying desire to serve and care for underserved populations. My clinical and research interests in many ways revolve around identifying disparities in healthcare access and healthcare outcomes. Foremost among these disparities are those due to race/ ethnicity and socioeconomics. As such, the population served by Highland Hospital, composed predominantly of indigent persons from the city of Oakland and the county of Alameda, is richly diverse, with many living at or below the poverty threshold. I quickly realized that I had always wanted to serve and care for this underserved population.

Despite a busy clinical service, my passion for clinical research remained undiminished, fueling my desire to continue building upon my clinical research interests. While the clinical research footprint was not as expansive as in a traditional academic environment, the ability to incorporate clinical research into one's practice is feasible with planning. The initial steps needed are to assess the available resources (i.e., personnel to help coordinate data, ability to extract clinical data, funding), what challenges impeded the development of a sustainable research program, and how to accomplish this within the allotted time. Furthermore, clinical research exists in many varieties, ranging from large clinical trials to observational cohort 
studies to smaller but more feasible case series or descriptive studies. The choice of study design must take into account not only the clinical question, but also the availability of resources and infrastructure required to successfully accomplish the project. Individuals interested in exploring a clinical question using their own clinical data should meet with their research advisors or senior faculty in order to develop a comprehensive plan. Prospective researchers should realize early that considerable time should be allocated for the planning process and for the gathering of clinical data using chart abstraction. Depending on the institution, some of these initial tasks can be supported by the bioinformatics or information technology departments. Other options include utilizing large registry data such as the Surveillance Epidemiology and End Results (SEER) database, the United Network for Organ Sharing (UNOS) transplant database, the National Health and Nutrition Examination Survey (NHANES), and the National Inpatient Sample (NIS). Whereas these datasets offer less granularity of data, the large sample size and pre-collected availability of data often make these good options for those with less time and resources to build their own clinical datasets. Although each type of study design or dataset has its own challenges, all data analyses do require basic skills in biostatistical analyses, emphasizing the importance of incorporating some exposure or formal instruction in clinical epidemiology.

Though having a background in clinical epidemiology and resources helps incorporate clinical research into one's career, equally important is the availability of mentorship, which can be present within one's division, within the larger department level, or even across different departments from individuals with specific skill sets. Developing a broad mentor base drawing from mentors encountered during medical school, residency, or fellowship, is also an effective strategy. Furthermore, one's current mentors and senior colleagues can provide networking opportunities to meet new faculty for potential mentorship.

Identifying funding to perform clinical research is challenging within an academic environment, and even more so at a non-traditional community-based program, where the concept of utilizing funding to "buy" protected time is not commonplace due to lack of familiarity with this concept. Nevertheless, funding can be used to support research assistance to help with study coordination, data extraction, and statistical analyses. While typical National Institutes of Health or career development-type funding may not be practical, smaller pilot grants given by foundations such as the American College of Gastroenterology or the American Cancer Society, or by health organizations such as the Kaiser Foundation Community Grants or the Patient-Centered Outcomes Research Institute are available. Furthermore, investigator-initiated research grants available from industry offer not only an alternative option for smaller-scale projects, but also offer valuable options such as obtaining investigational drugs and performing specialized assays.

In summary, incorporating clinical research into one's career is not easy, but definitely achievable. The keys to success are: (1) developing a broad base of mentors and advisors; (2) developing a fundamental skill set in clinical epidemiology; (3) developing a clear understanding of what degree of research you want to incorporate into your current practice; (4) accurately assessing the current resources available to you and the challenges you face to achieve your research goals; (5) working with your leadership to help identify opportunities and resources for you to accomplish these goals, and (6) never losing the passion that fuels your excitement about clinical research.

\section{References}

1. Tong CW, Ahmad T, Brittain EL, et al. Challenges facing early career academic cardiologists. $J$ Am Coll Cardiol. 2014;63:2199-2208.

2. Cadman EC. The academic physician-investigator: a crisis not to be ignored. Ann Intern Med.. 1994;120:401-410. 